\title{
Long-term spot topography and multi-band flare analysis of active ultra-fast rotator LO Peg
}

\author{
Shashi Bhushan Pandey ${ }^{1 *}$, Jeewan C. Pandey ${ }^{1}$, Subhajeet Karmakar ${ }^{1}$, Igor S. \\ Savanov $^{2}$ \\ ${ }^{1}$ Aryabhatta Research Institute of Observational Sciences, Nainital, India 263001 \\ E-mail: shashiearies.res.in \\ ${ }^{2}$ Institute of Astronomy, Russian Academy of Sciences, ul. Pyatniskaya 48, Moscow 119017, \\ Russia
}

\begin{abstract}
We present analyses of optical photometric and X-ray observations of the star LO Peg. Nearly 26 yrs of optical observations show that LO Peg is a periodic variable with a photometric period of $0.422853 \pm 0.000005 \mathrm{~d}$. Light curves observed at 47 epochs in V-band show changes in minima, amplitude and shape indicating that the variability is due to the presence of surface inhomogeneities. The surface coverage of spots was found to be in the range of 9-26\%. Most of the time, the spots were resolved as two active longitudes. The corona of LO Peg consists of a two temperature plasma of $3.24 \times 10^{6} \mathrm{~K}$ and $11.95 \times 10^{6} \mathrm{~K}$. The average X-ray luminosity in the $0.3-10.0 \mathrm{keV}$ energy band was found $3.56 \times 10^{29} \mathrm{erg} \mathrm{s}^{-1}$. Flaring features in optical V-band with flare energies of the order of $10^{33} \mathrm{erg}$ were also noticed.
\end{abstract}

Swift: 10 Years of Discovery

2-5 December 2014

La Sapienza University, Rome, Italy

${ }^{*}$ Speaker. 


\section{Introduction}

Magnetic activities of cool ultra fast rotators (UFR; vsini $>40 \mathrm{~km} \mathrm{~s}^{-1}$ ) are important as they are in the saturation or supersaturation $\left(\mathrm{L}_{\mathrm{X}} / \mathrm{L}_{\mathrm{bol}} \leq 10^{-3.0}\right)$ regions of rotation activity diagram (e.g., Feigelson et al. 2003). Speedy Mic ( $\left.\mathrm{P}=0.3804 ; \mathrm{L}_{\mathrm{X}} / \mathrm{L}_{\mathrm{bol}}=10^{-2.27}\right)$, AB Dor $(\mathrm{P}=0.5139$; $\left.\mathrm{L}_{\mathrm{X}} / \mathrm{L}_{\mathrm{bol}}=10^{-2.93}\right)$, HK Aqr $\left(\mathrm{P}=0.4312 ; \mathrm{L}_{\mathrm{X}} / \mathrm{L}_{\mathrm{bol}}=10^{-3.37}\right)$ and LO Peg $\left(\mathrm{P}=0.42312 ; \mathrm{L}_{\mathrm{X}} / \mathrm{L}_{\mathrm{bol}}=10^{-3.0}\right)$ are some famous examples of late-type active UFRs. Some of them are very active, e.g. AB Dor is showing $\sim 4$ flares per rotation period (Karmakar \& Pandey 2015). However, others are relatively less active. Dark spots on the surface, flares from radio to X-rays are common magnetic activities in these cool UFRs (Strassmeier 2009). Stellar magnetic activities in these stars are not well understood due to the lack of spatial information. Therefore, indirect techniques e.g. Doppler imaging (Vogt \& Pernoid 1983) and inversion techniques (Savanov \& Strassmeir 2008) are useful. The high resolution and high signal-to-noise ratio spectroscopic observations with a good phase coverage as required for Doppler imaging are limited, therefore, long-term traditional photometric observations are important to understand the active region evolution and the stellar activity cycles (e.g. Olah et al. 1997; Pandey et al. 2005, Savanov 2010, Patel et al. 2013).

In this paper, we present analyses of long term optical and $S$ wift $X$-ray observations of an UFR LO Peg. It is a young, K5V-K8V type and member of AB Doradus moving group (Zuckerman et al. 2004). Presence of strong flaring activity was identified by Jeffries et al.(1994) and Eibe et al. (1999) from $H_{\alpha}$ and He I D3 observations. Pandey et al. (2009) showed strong modulation in broad band linear polarization and suggested that it could be the result of scattering of an anisotropic stellar radiation field by an optically thin circumstellar envelope or scattering of the stellar radiation by prominence-like structures. The previous X-ray observations LO Peg from ROSAT estimated its X-ray luminosity to be $10^{29.7} \mathrm{erg} \mathrm{s}^{-1}$ (Pandey et al . 2005). However, present observations span over $\sim 4$ years to study the long term observations.

\section{Observations and data reduction}

LO Peg was observed with Swift satellite for several occasions from April 30, 2008 to July 2, 2012 (Obs IDs:00037810 ; PI S. B. Pandey \& J. C. Pandey). The observations were made in soft X-ray band (0.3-10.0 keV) with X-Ray Telescope (XRT; Burrows et al. 2005) in conjunction with UV/Optical Telescope (UVOT; Gehrels et al. 2004) in UV bands (170-650 nm). The XRT exposure time of LO Peg ranges from $0.3 \mathrm{ks}$ to $5.0 \mathrm{ks}$. X-ray light curves and spectra of LO Peg were accumulated from on-source counts obtained from circular regions on the sky centered on the X-ray peaks. The X-ray light curves and spectra for the source and background were extracted using the XSELECT package for the $0.3-10.0 \mathrm{keV}$ energy band of both the cases. The background was accumulated from several neighboring regions at nearly the same offset as the program star.

We also observed LO Peg several times between October 25, 2009 and December 18, 2013 in Johnson U, B, V and R photometric bands with 1.04-m telescope of ARIES, Nainital using $2 k \times 2 k$ CCD camera, which covers $\sim 13^{\prime} \times 13^{\prime}$ of the sky. We make use of the various data in $\mathrm{V}$ band from literature (Jeffries et al. 1994, Pandey et al. 2005, 2009, Tas 2011) and archive. The archival data were taken from Hipparcos (Perryman et al. 1997), SuperWasp (Street et al. 2003, Pollacco et al. 


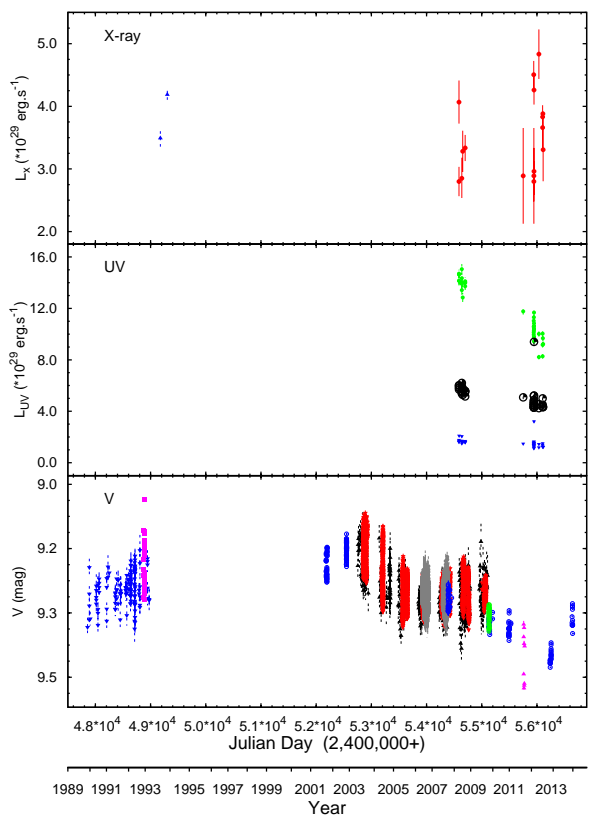

(a) Multi-wavelength light curve

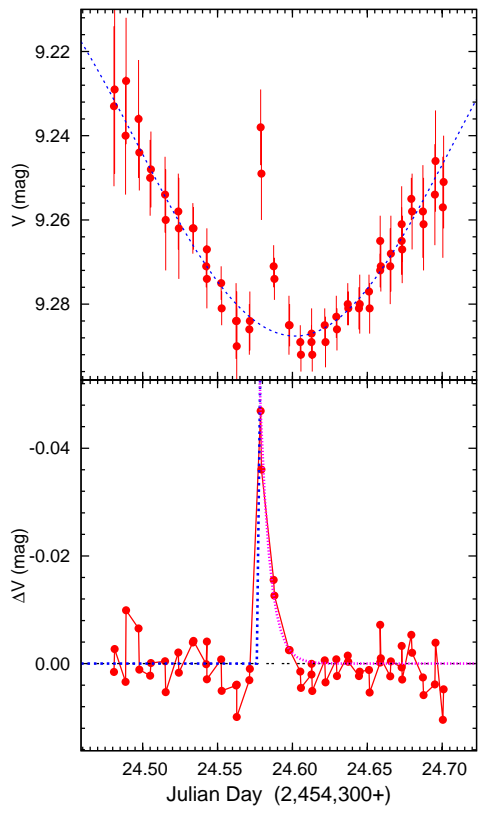

(b) Flare

Figure 1: (a) Multiwavelength X-ray, UV and optical light curves of LO Peg. (b) V-band light curve showing flaring feature, the bottom panel shows detrended light curve.

2006) and All Sky Automated Survey (ASAS; Pojmansky 2002) observations. Optical broad band data were reduced using the standard tasks in IRAF.

\section{Analysis and Results}

\subsection{Multi-wavelength light curves and Inhomogeneity map}

The long term multiwavelength light curve of LO Peg is shown in Figure 1 (a). The long as well as short term variability was clearly observed in the V-band light curve. The long term variability in X-ray and UV band was also noticed. The periodogram analysis of $\mathrm{V}$ band data shows photometric period of LO Peg to be $0.422853 \pm 0.000005$ days. In order to see the short term variability/fluctuations, we have manually checked V-band light curve of each night observations. Apart from the short term rotational modulation, we have identified the flaring like feature in the V-band light curves. Top panel of Figure 1(b) shows the example of such flare like feature, where V-band light was enhanced suddenly and decayed gradually. In order to extract the flaring features only, we have removed the rotational modulation by fitting the sinusoidal function. The lower panel of Figure 1(b) shows the detrended light curve, where flaring feature is clearly noticed. We found 12 such flares from V-band data and flare frequency was derived to be $\sim 1$ flare per three days. Assuming that the star is a blackbody radiator, the flare energy is calculated as the excess energy 
14

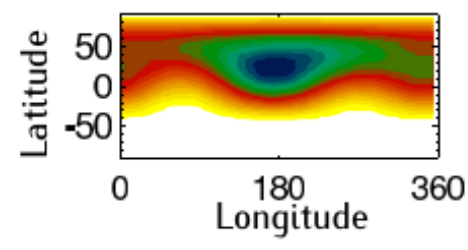

14

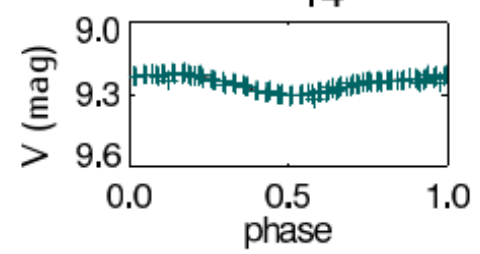

(a) $\mathrm{HJD}=2453572.99$

15

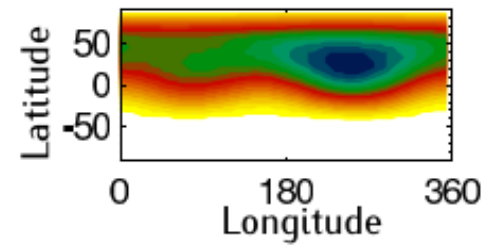

15

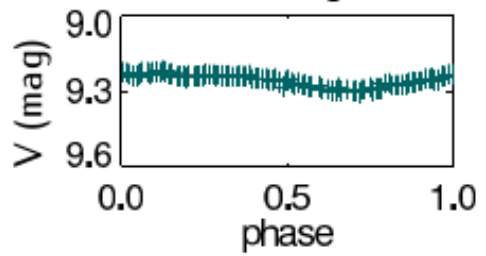

36

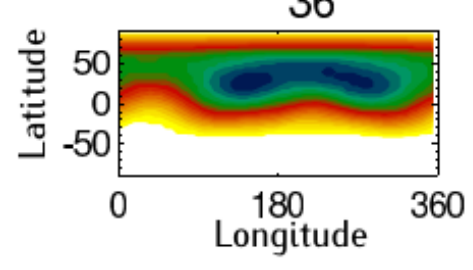

(b) $\mathrm{HJD}=2453617.51$

(c) $\mathrm{HJD}=2454372.90$

Figure 2: Surface inhomogeneity map (left panel) and corresponding phase folded V-band light curve (right panel) at three epochs of HJD= 2453572.99, 2453617.51 and 2454372.9.

released at the time of flare. Flare energies of these flares were derived to be of the order of $10^{33}$ erg.

In order to generate the surface map of LO Peg, we used the light-curve inversion code (iPH) developed by Savanov \& Strassmeier (2008) and Savanov (2009). The entire light curve of all observations were divided into 47 sets without gaps on the curve on the phase diagram and without changes of its shape. The resultant light curves were not constant, and showed the change in shape, amplitude and minimum of light. The amplitude of these light curves were in the range of 0.06 0.20 mag. Each individual light curve was analysed using the iPH code. We adopt the temperature of LO Peg photosphere to be $\sim 4500 \mathrm{~K}$ and and the temperature of the spots to be $750 \mathrm{~K}$ lower than surface temperature (Piluso et al. 2008). We used the data from the Kurucz's model grid in our computations. Reconstruction of the temperature-inhomogeneity maps was carried out for angle of inclination of 45 degrees. Figure 2 shows the inhomogeneity map at three mean epochs. Most of the time, spots were concentrated into two groups at two different longitudes, where the differences of longitude were not constant. The total areas covered by group of spots were in the range of 9$26 \%$ of the total visible stellar surface. The spot area in LO Peg is very similar to that found in other K-type stars like V1147 Tau (Patel et al. 2013, Savanov 2010) and are more than that for the evolved RS CVn-type binaries. 


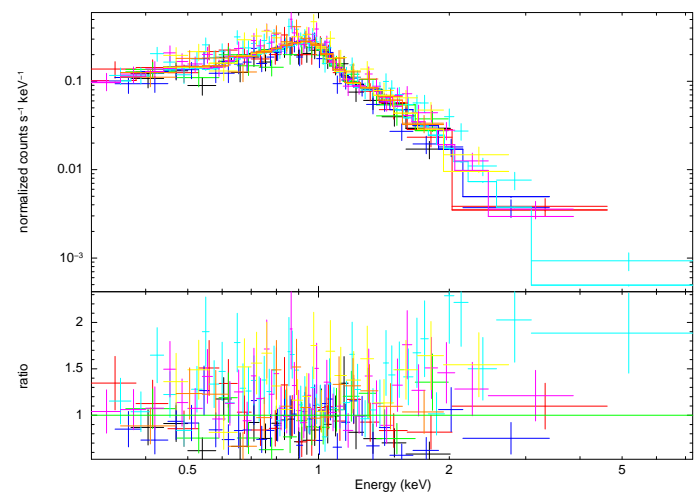

Figure 3: Background subtracted X-ray spectra of LO Peg with the best fit two temperature plasma model apec. The bottom panel shows the residual in terms of ratio.

\subsection{X-ray spectra}

Figure 3 shows the background subtracted X-ray spectra of LO Peg as observed from Swift XRT. For all set of observations, the X-ray spectra of LO Peg were fitted with two temperature plasma model APEC (Smith et al. 2001), with variable elemental abundances, Z. The interstellar hydrogen column density $\left(\mathrm{N}_{H}\right)$ was left free to vary. All the parameters were found to be constant within $1 \sigma$ level for all observations; therefor, these parameters were kept fixed at the average value to determine the unabsorbed flux. The variation of unabsorbed flux is shown in the top panel of Figure 1. The derived value of temperatures and corresponding emission measures were $0.28 \pm$ $0.04 \mathrm{keV}$ and $3.1 \pm 0.09 \times 10^{52} \mathrm{~cm}^{-3} \mathrm{~cm}^{3}, 1.03 \pm 0.05 \mathrm{keV}$ and $4.6 \pm 0.06 \times 10^{52} \mathrm{~cm}^{-3} \mathrm{~cm}^{3}$, respectively. Abundances were found to be $0.13 \pm 0.02$ solar unit. These values are similar to those derived from the ROSAT observations (Pandey et al. 2005).

\section{Summary}

We have carried out analyses of long term optical and X-ray observations of an active UFR LO Peg. The variable shapes and amplitudes of light curves of LO Peg at 47 epochs indicate the temperature inhomogeneities on its surface. A modeling of these observations reveals that the stellar surface of LO Peg is spotted up to 26 per cent, which is very similar to that found in K-type stars. A total of 12 flares during the observations were found indicating $\sim 1$ flare per eight rotation of LO Peg. The corona of LO Peg was well represented by two temperature plasma of 0.28 and $1.03 \mathrm{keV}$. The X-ray luminosity of LO Peg over the $\sim 4$ years of the observations were found to be constant with an average value of $3.56 \pm 0.11 \times 10^{29} \mathrm{erg} \mathrm{s}^{-1}$. Detail study of multi-wavelength data will be published elsewhere (Karmakar et al. 2015)

\section{References}

[1] Burrows David N. et al., The Swift X-Ray Telescope, SSRv, 120, (2005), 165.

[2] Eibe M. T., Byrne P. B., Jeffries R. D., Gunn, A. G., Evidence for large-scale, global mass inflow and flaring on the late-type fast rotator BD22 4409, A\&A, 341, (1999), 527. 
[3] Fiegelson Eric D. et al., X-rays in the Orion Nebula Cluster: Constraints on the origins of magnetic activity in pre-main sequence stars, ApJ, 584, (2003), 911.

[4] Gehrels N. et al., The Swift Gamma-Ray Burst Mission, ApJ, 611, (2004) 1005.

[5] Jeffries R. D., Byrne P. B., Doyle J. G., Anders G. J., James D. J., Lanzafame A. C., BD+22DEG4409 a Rapidly Rotating Low-Mass Member of the Local Association, MNRAS, 270, (1994), 153.

[6] Karmakar, S. \& Pandey J. C., XMM-Newton observations of AB-Dor : X-ray flares, in preparation (2015).

[7] Karmakar, S. et al., LO Peg : spot-topographic evolution, surface differential rotation and flares, to be submitted to ApJ, (2015)

[8] Olah K., Kovari Zs., Bartus J., Strassmeier K. G., Hall D. S., Henry G. W., Time-series photometric SPOT modeling. III. Thirty years in the life of HK Lacertae, A\&A, 321, (1997), 811.

[9] Pandey J. C., Singh K. P., Drake S. A., Sagar R., Optical and X-Ray Studies of Chromospherically Active Stars: FR Cancri, HD 95559, and LO Pegasi, AJ, 103, (2005) 1231.

[10] Pandey J. C., Medhi Biman J., Sagar R., Pandey A. K., LO Pegasi: an investigation of multiband optical polarization, MNRAS, 396, (2009), 1004.

[11] Patel M. K., Pandey, J. C., Savanov I. S., Prasad V., Srivastava, D. C., Study of photospheric, chromospheric and coronal activities of V1147 Tau, MNRAS, 430, (2013) 2154.

[12] Perryman M. A. et al., The HIPPARCOS Catalogue, A\&A, 323, (1997), L49.

[13] Piluso N., Lanza A. F. et al., Doppler imaging of the young late-type star LO Pegasi (BD+22Ârr4409) in 2003 September, MNRAS, 387, (2008), 237.

[14] Pollacco, D. L., Skillen, I., Collier Cameron, A., et al., The WASP Project and the SuperWASP Cameras, PASP, 118, (2006), 1407.

[15] Savanov I. S., Spots and active longitudes on the star V815 Her , Astron. Rep., 53, (2009), 941.

[16] Savanov I. S., Magnetic activity of the star Corot-Exo-2a, Astron. Rep., 54, (2010), 437.

[17] Savanov I. S. \& Strassmeier K. G.,Surface imaging with atomic and molecular features. I. A new inversion technique and first numerical tests, $A \& A, \mathbf{4 4 4},(2005), 931$.

[18] Savanov I. S. \& Strassmeier K. G.,Light-curve inversions with truncated least-squares principal components: Tests and application to HD 291095 = V1355 Orionis, Astron. Nachr., 329, (2008), 364.

[19] Strassmeier K. G., Starspots, ARA\&A, 17, (2009), 251.

[20] Smith R. K., Brickhouse N. S., Liedahl D. A., Raymond J. C., Collisional Plasma Models with APEC/APED: Emission-Line Diagnostics of Hydrogen-like and Helium-like Ions, ApJ, 556, (2001), L91.

[21] Street R. A. et al., Searching for planetary transits in the field of open cluster NGC 6819 - I, MNRAS, 340, (2003), 128

[22] Tas, G.,Long-term photometry of the ultra-fast rotator LO Peg, AN, 332, (2011), 57.

[23] Vogt S. S., Penrod G. D., Doppler Imaging of spotted stars - Application to the RS Canum Venaticorum star HR 1099, PASP, 95, (1983), 565.

[24] Zuckerman B., Song I., Bessel M. S., The AB Doradus Moving Group, ApJ, 613, (2004), L65 


\section{Acknowledgements}

This work was done under the Indo-Russian DST-RRBR project reference INT/RUS/RFBR/P167 (for India) and Grant RFBR Ind_a 14-02-92694 (for Russia). We acknowledge the Swift data archive and the UK Swift Science Data Center. The authors also acknowledge the Swift team to provide the observing time under the fill-in targets. 